\title{
Microanalysis of Autogenous Healing Products in Engineered Cementitious Composites (ECC)
}

\author{
Aaron Richard Sakulich, ${ }^{*}$ Lili Kan, ${ }^{* *}$ and Victor C. Li* \\ * Department of Civil and Environmental Engineering, University of Michigan, 2350 Hayward St., \\ Ann Arbor, MI 48109-2125 \\ ** Key Laboratory of Advanced Civil Engineering Materials of Education Ministry, Tongji \\ University, Shanghai, China
}

Cracking is a major problem in cementitious systems. It can be caused by loading, creep, shrinkage, chemical attack, or cycles of thermal expansion and contraction. Whatever the cause, cracking inexorably leads to lowered durability in the form of reduced mechanical properties and inability to protect reinforcing steel. The ability of a material to self-heal, that is to heal cracks without resort to external media, has been termed autogenous healing [1]. In a perfect system, autogenous healing would occur automatically upon the creation of a crack and would not require any external influence. The crack would be both sealed (reducing flow of liquids through the material) and, more importantly, healed, (restoring the material's mechanical properties). A cementitious material capable of robust autogenous healing would have a substantially higher durability, longer life, lower overall cost, and would be more environmentally friendly.

Autogenous healing of cementitious materials occurs due to one of two mechanisms: either the hydration of unreacted cement particles or the carbonation of portlandite crystals, both of which are exposed during cracking. A number of studies on ordinary cement have shown the production of calcite to be the most important, and sometimes only, mechanism at work. Autogenous healing, via these mechanisms, occurs only in the presence of water, and only in very fine cracks below roughly $50 \mu \mathrm{m}$ wide. The autogenic healing capabilities of ordinary cementitious materials are poor, as the crack size cannot be controlled or predicted, and is often far too large for autogenous healing to occur (fig. 1a).

Engineered Cementitious Composites (ECCs) are a class of ultra-ductile, micromechanically designed, fiber-reinforced cementitious composites [2]. The high ECC tensile strain capacity of above $3 \%$ is due to distributed microcracking produced by low loadings $(<2 \%)$ of polymer fibers (fig. 1b). These microcracks are small enough to be autogenously healed, and can be custom tailored depending on application.

Much work on autogenous healing in ECC has focused on mechanical properties. It is known that healing products not only seal cracks, but substantially improve stiffness and ultimate tensile strength upon reloading $[3,4]$. Chemical characterization of the healing products themselves, however, has received limited attention.

A number of microanalysis techniques, such as FTIR, Raman, and energy dispersive spectroscopies complimented by x-ray diffraction have been used to characterize self healing products in ECC. The data show that the product is a mixture of both hydrated cement and calcite. The FTIR results in particular suggest that the calcite is not being produced by carbonation of portlandite, but rather by $\mathrm{CO}_{3}{ }^{2-}$ ions in solution stripping calcium from the calcium silicate hydrate $(\mathrm{C}-\mathrm{S}-\mathrm{H})$ phase of the 
cement. Both optical and electron microscopy (fig. $2 \mathrm{a}$ and $2 \mathrm{~b}$, respectively) have shown that the crack-bridging fibers provide important nucleation sites for healing products, leading to a complex micro-composite. Finally, both nanoindentation and in-situ SEM tensile tests were used to characterize the mechanical properties of the healing product.

Although more work is needed, the extant data clearly show that ECC have a capacity for robust autogenic healing, which is not found in ordinary cementitious systems. This healing capacity is one of a number of properties that make ECC an extremely durable, environmentally friendly material.
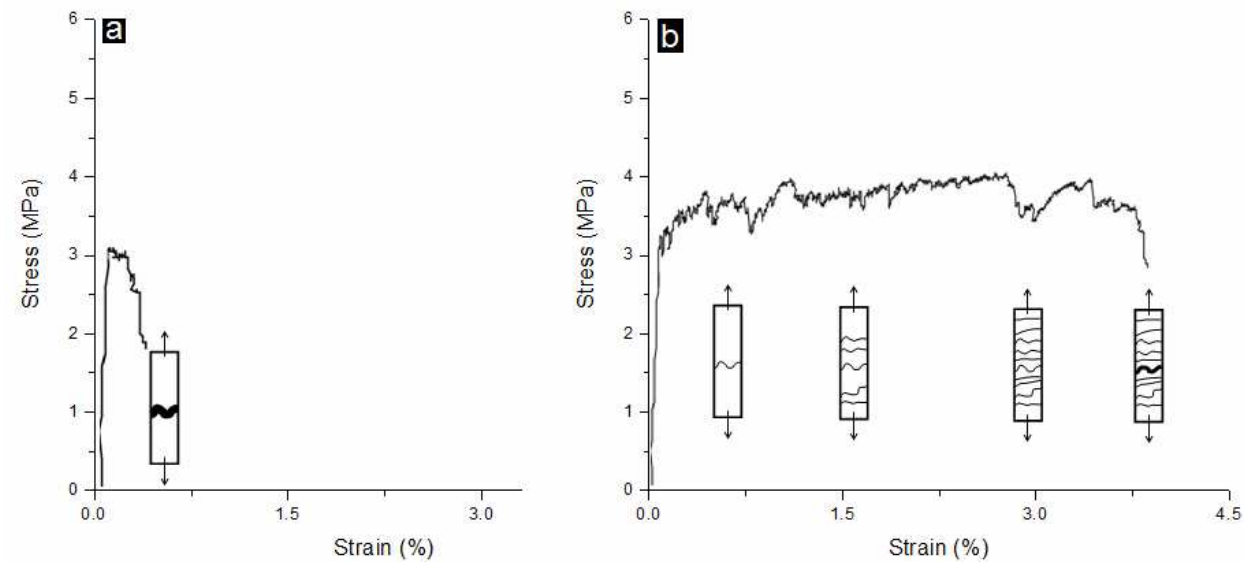

FIG 1. The difference between ordinary cementitious systems (a) which fail by major cracking at very low strains, and ECC (b) which fail via distributed microcracking at ultra high strains.
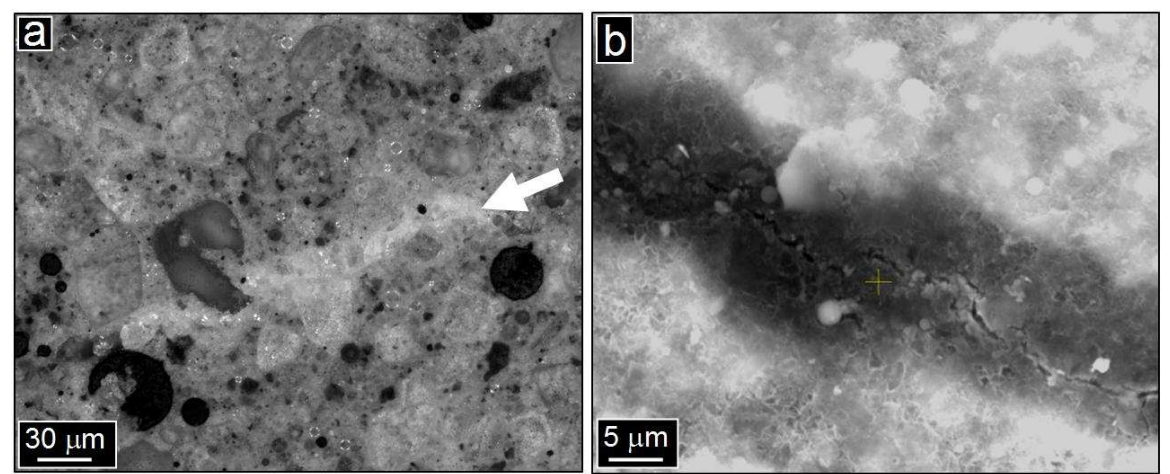

FIG 2. Optical (a) and SEM (b) micrographs of autogenous healing products in ECC (white phase with arrow, and dark phase, respectively). These products were shown to be a mixture of calcite and hydrated cement

References

[1] K.R. Lauer et al., J. Amer. Concrete Inst., 27 (1956) 1083.

[2] V.C. Li, J. Adv. Concrete Tech., 1 (2003) 215.

[3] Y. Yang et al., Cem. and Concrete Res. 39 (2009) 382

[4] V.C. Li et al., Self Healing in Concrete Materials in Self Healing Materials: An Alternative Approach to 20 centuries of Materials Science, Springer Science, Dordrecht, 2007. 